\title{
Quiinaceae do Nordeste
}

Quiinaceae from Northeast Brazil

Anderson Alves-Araújo ${ }^{1,2}$, Maria Fátima Araújo Lucena ${ }^{1} \&$ Marccus Alves $^{1}$

\begin{abstract}
Resumo
Quiinaceae está representada no Nordeste do Brasil por quatro espécies e dois gêneros: Lacunaria crenata, Quiina cruegeriana, Q. florida e Q. glazovii. Na Região, ocorrem exclusivamente em floresta atlântica, com preferência por ambientes mais úmidos e preservados. São registrados casos de endemismos na floresta atlântica ( $Q$. glazovii) e disjunção entre as florestas amazônica e atlântica ao norte do rio São Francisco $(Q$. cruegeriana e Q. florida). São apresentados comentários sobre distribuição geográfica, habitats, caracteres diagnósticos e ilustrações, além de um novo sinônimo.

Palavras-chave: Brasil, florística, floresta atlântica, Quiina, Lacunaria.
\end{abstract}

\begin{abstract}
Quiinaceae is represented in Northeast Brazil by four species and two genera: Lacunaria crenata, Quiina cruegeriana, Q. florida, and Q. glazovii. They occur in the Atlantic forest, clearly preferring wet, preserved habitats. A new synonym is presented as well as comments on geographic distribution, habitats, and diagnostic characters.
\end{abstract}

Key words: Brazil, floristics, Atlantic rainforest, Quiina, Lacunaria.

\section{Introdução}

Quiinaceae (Eurosidea I, sensu APG - Stevens 2001) é uma família monofilética (Schneider et al. 2002), endêmica dos Neotrópicos, com quatro gêneros e cerca de 40 espécies, sendo a maioria ocorrente na floresta amazônica (Zizka \& Schneider 2004; Souza \& Lorenzi 2008). Os quatro gêneros (Froesia Pires, Lacunaria Ducke, Quiina Aubl. e Touroulia Aubl.) ocorrem no Brasil, com aproximadamente 30 espécies registradas (Barroso et al. 1978; Rocha \& Secco 2004; Souza \& Lorenzi 2008). Apesar da maior diversidade taxonômica nas florestas ao norte do país, foram encontradas amostras da floresta atlântica em acervos locais. Para o domínio Atlântico (sensu Fiaschi \& Pirani 2009), com exceção da Flora brasiliensis (Engler 1888), os estudos com a família estão restritos às Regiões Sudeste (Souza \& Bianchini 2001; 2007) e Sul (Reitz 1965; 1972), não tendo sido citada para o Nordeste do Brasil por Barbosa et al. (2006). No entanto, Grilo et al. (2006), Barreto et al. (2006) e Ferraz \& Rodal $(2006,2008)$ citam a família como parte integrante de suas listas florísticas, apesar de apresentarem imprecisão na identificação das amostras.

Os representantes da família são tipicamente árvores de pequeno e médio porte, com folhas simples ou compostas pinadas, opostas ou verticiladas e estípulas interpeciolares. Tais características remetem, especialmente quando em estado vegetativo, a Rubiaceae. As flores são unissexuadas ou bissexuadas, actinomorfas, diclamídeas, polistêmones e com ovário súpero. Os frutos são bagas, por vezes deiscentes, e a superfície apresenta estrias longitudinais bem marcadas. Segundo Costa \& Amaral (1999), os frutos são dispersados por animais e em algumas regiões da Amazônia a madeira é empregada na construção civil e na movelaria artesanal.

Durante os estudos para a flora do Nordeste, foram constatadas deficiências nas coleções de Quiinaceae em diversos herbários regionais, particularmente no que se refere à identificação taxonômica das amostras. Em face deste cenário, objetiva-se com este trabalho propiciar um melhor

\footnotetext{
${ }^{1}$ Universidade Federal de Pernambuco, Dept. Botânica, Lab. Morfo-Taxonomia Vegetal, Av. Moraes Rego 1235, Cidade Universitária, 50670-901, Recife, PE, Brasil.

${ }^{2}$ Autor para correspondência: sapotae@gmail.com
} 
conhecimento da família na Região, além de subsidiar a Lista de Espécies da Flora do Brasil.

\section{Materiais e Métodos}

As amostras analisadas para este estudo foram obtidas através de coletas em diversos estados do Nordeste, assim como provenientes dos acervos botânicos (ASE, CEPEC, EAC, HST, IPA, JPB, MAC, PEUFR, RB, TEPB, UFP e UFRN; siglas de acordo com Thiers 2009). Foram utilizadas bibliografias específicas para identificação e consulta às imagens do material tipo disponibilizado online. As amostras coletadas estão depositadas no herbário UFP.

\section{Resultados e Discussão}

Foram catalogadas quatro espécies de Quiinaceae para o Nordeste: Lacunaria crenata (Tul.) A. C. Sm., Quiina cruegeriana Griseb., $Q$. florida Tul. e Q. glazovii Engl. Elas estão restritas à floresta atlântica, desde o sul da Bahia até o norte de Pernambuco, ocorrendo em subosque úmido, entre 100 a 600 m de altitude. Quiina glazovii é endêmica da floresta atlântica, ao passo que as demais espécies apresentam distribuição disjunta entre as florestas atlântica e amazônica. São comumente arvoretas a árvores de pequeno porte (até $18 \mathrm{~m}$ alt.), geralmente formando populações com poucos indivíduos. Diferenciam-se facilmente de Rubiaceae pelo formato das estípulas associado a caracteres reprodutivos como as flores dialipétalas, por vezes unissexuadas, e o ovário súpero. A cor esbranquiçada do tronco externamente e avermelhada internamente auxiliam o reconhecimento do grupo no campo. A filotaxia, o comprimento e a pilosidade da lâmina foliar e a forma e o comprimento das estípulas são caracteres importantes para o reconhecimento das espécies.

\section{Chave para identificação de Quiinaceae do Nordeste}

1. Plantas dióicas com folhas verticiladas 1. Lacunaria crenata

1'. Plantas androdióicas com folhas opostas Quiina

2. Folhas $\geq 16 \mathrm{~cm}$ compr., estípulas ovadas, inflorescências $\geq 12 \mathrm{~cm}$ compr. 4. Q. glazovii

2. Folhas $<16 \mathrm{~cm}$ compr., estípulas nunca ovadas, inflorescências $<12 \mathrm{~cm}$ compr.

3. Nervuras intersecundárias conspícuas, estípulas filiformes a lineares, densamente pilosas .

2. Q. cruegeriana

3'. Nervuras intersecundárias inconspícuas, estípulas elípticas a raramente lanceoladas, glabras 3. Q. florida

1. Lacunaria crenata (Tul.) A.C. Sm., Trop. Woods 58: 31. 1939. Quiina crenata Tul., Ann. Sci. Nat. Bot., sér. 3, 11: 163. 1849.

Fig. 1a-b

Lacunaria decastyla (Radlk.) Ducke, Arch. Jard. Bot. Rio de Janeiro 5: 169. 1930.

Lacunaria pauciflora Ducke, Arch. Jard. Bot. Rio de Janeiro 5: 169. 1930.

Arvoretas a árvores dióicas, com 7-18 m alt. e registro no domínio Atlântico limitado a poucas amostras provenientes do sul da Bahia e Minas Gerais. Segundo Rocha \& Secco (2004) e Schneider \& Zizka (2004), além de ocorrer na Mata Atlântica, Lacunaria crenata é amplamente distribuída na região amazônica do Brasil (AC, AM, AP, PA, RO), Colômbia, Equador, Guiana, Guiana Francesa, Peru, Suriname e Venezuela.

Dentre as espécies analisadas, pode ser facilmente reconhecida pela folhas verticiladas (opostas nas espécies de Quiina), glabras, com 10-
15 cm compr., margem ondulada, serreada a crenada, nervuras intersecundárias geralmente inconspícuas e estípulas lineares a subuladas, glabras; inflorescências com 8-10 cm compr e bagas ovóides a cônico-elipsóides.

Material examinado: BAHIA: Itacaré, 7.V.1968, R.P. Belém 3511 (CEPEC). Porto Seguro, estrada para Eunápolis, 13.X.1983, fr., G. Martinelli \& T. Soderstrom 9636 (CEPEC, RB). Prado, rod. BA-284, Prado a Itamaraju, 18.IX.1978, fr., S.A. Mori et al. 10637 (CEPEC). Santa Cruz Cabrália, Estação Ecológica do Pau-Brasil, 28.XI.1979, fl., S.A. Mori et al. 13043 (CEPEC, MG). Una, fazenda São Rafael, 14.XII.1968, fl., T.S. Santos 316 (CEPEC, MG).

2. Quiina cruegeriana Griseb., Fl. Brit. W. I.: 106. 1864.

Fig. 1c-d Quiina pernambucensis Pires \& AndradeLima nom. nud., syn. nov.

Arvoretas androdióicas, com 3-8 m alt. e registro para o Ceará, Pernambuco e Alagoas. De 



Figura 1 - a-b. Lacunaria crenata A. C. Sm. - a. detalhe do ramo, evidenciando a filotaxia verticilada e as estípulas (Mori 13043); b. fruto (Martinelli 9636). c-d. Quiina cruegeriana Griseb. (Ferraz 609) - c. ramo, mostrando filotaxia oposta; d. detalhe do ápice do ramo, mostrando as estípulas filiformes e densamente pilosas. e. $Q$. florida Tul. (Oliveira 1267) - detalhe do nó, mostrando as estípulas. f-g. Q. glazovii Engl. (T. S. Santos 1053) - f. ramo com inflorescências; g. detalhe do nó, mostrando as estípulas.

Figure 1 - a-b. Lacunaria crenata A. C. Sm. - a. detail of stem, showing verticillate leaves (Mori 13043); b. fruit (Martinelli 9636). c-d. Quiina cruegeriana Griseb. (Ferraz 609) - c. detail of stem, showing the opposite leaves; d. detail of stem apex, showing the filiform hairy stipules. e. Quiina florida Tul. (Oliveira 1267) - detail of node, showing the stipules. f-g. Quiina glazovii Engl. (Santos 1053 ) - f. shoot with inflorescences; g. detail of node, showing stipules. 
acordo com Schneider \& Zizka (2004), Quiina cruegeriana ocorre no Brasil, Caribe, Costa Rica, Equador, Guiana Francesa, Panamá, Peru, Suriname, Trinidad e Tobago e Venezuela, em altitudes entre 300 e 2.000 m; na Mata Atlântica, foi registrada apenas para áreas ao norte do rio São Francisco.

Quiina cruegeriana é reconhecida pelos tricomas de coloração dourado-ferrugínea na face abaxial das folhas e nos ramos jovens, folhas com 10-15 cm compr., margem levemente revoluta, nervuras intersecundárias conspícuas e estípulas filiformes a lineares, densamente pilosas; inflorescências com 6-10 cm compr., composta por flores de coloração amarelo-intensa e odoríferas; e bagas elipsóides a globóides.

Ferraz \& Rodal $(2006,2008)$ citaram Quiina pernambucensis em inventários florestais, porém a espécie não conta com diagnose efetivamente publicada. O exame do material testemunho e a consulta de bibliografia específica revelaram tratarse de amostras de $Q$. cruegeriana com folhas maiores e com maior densidade de tricomas, o que representa apenas uma variação morfológica da espécie.

Material examinado: ALAGOAS: Barra de Santo Antônio, D. Andrade-Lima 67-9038 (IPA). Ibateguara, Coimbra, Grotão do Dudé, 12.III.2003, fr., M. Oliveira \& A.A. Grilo 1303 (UFP). Murici, APA de Murici, fazenda Poço D'Antas, 5.VI.1993, fl., R.P. Lyra-Lemos 2781 (MAC, UFP); Reserva Biológica de Murici, 10.II.2009, fl., A. Alves-Araújo et al. 1127 (UFP). CEARÁ: [Pacatuba], Serra da Patuba [Serra da Pacatuba], 10.III.1968, fl., D. Andrade-Lima 68-45 (MO); Serra da Aratanha, Sítio Pitiguari, 2.X.1979, fr., A.J. Castro \& P. Martins (EAC-4122). PERNAMBUCO: Cabo, Gurjaú, margem direita da represa, 27.II.1962, fl., J.M. Pires \& D. Andrade-Lima 62-4042 (IPA); Reserva Estadual de Gurjaú, Mata do Xangô, 6.XI.2003, fl., J.B.S. Oliveira et al. 17(UFP); Mata do Cuxió, 28.X.2003, fl., A. Viana et al. 187 (UFP). Escada, Usina Massuaçu, 6.IV.1973, fr., D. Andrade-Lima 73-7311 (IPA). Engenho Conceição, 21.V.1968, fr., O.C. Lira 68-243 (IPA, TEPB); 10.VI.1967, fr., D. Andrade-Lima 67-9039 (IPA). Jaqueira, RPPNFrei Caneca, Mata do Quengo, 23.IV.2003, fr., J. Leal et al. 28 (UFP); 23.IV.2003, fr., $J$. Leal et al. 41 (UFP). São Vicente Férrer, Mata do Estado, 9.III.1998, fr., A. Laurênio et al. 822 (PEUFR); 12.II.1999, fl., E.M.N. Ferraz et al. 609 (CEPEC, PEUFR, UFRN). Timbaúba, Engenho Água Azul, 26.II.1969, fr., D. AndradeLima 69-5530 (IPA, JPB). Vicência, Engenho Jundiá, 5.I.1967, fl., D. Andrade-Lima 67-9037 (IPA).

3. Quiina florida Tul., Ann. Sci. Nat. Bot. sér. 3, 11: 167. 1849.

Fig. 1e

Quiina poeppigiana Tul., Ann. Sci. Nat. Bot., sér. 3, 11: 161. 1849.

Arvoretas androdióicas, com 4-8 m alt. e registro para Alagoas e Pernambuco. No Brasil, é amplamente distribuída na floresta amazônica (AC, AM, AP, PA, RO), sendo aqui mais um registro de disjunção com a Mata Atlântica ao norte do Rio São Francisco. Segundo Schneider \& Zizka (2004), ocorre ainda na Bolívia, Colômbia, Equador, Peru e Venezuela, em altitudes entre 100 e $200 \mathrm{~m}$.

Quiina florida apresenta folhas e ramos jovens glabros, folhas com 8-14 cm compr., margem levemente ondulada, nervuras intersecundárias inconspícuas e estípulas elípticas a raramente lanceoladas, rígidas a foliáceas, glabras; inflorescências com 8-12 cm compr., compostas por flores de coloração amarelo-intensa e odoríferas; e bagas elipsóides a globóides. Destaca-se pelas folhas e estípulas glabras (pilosas em $Q$. cruegeriana) e estípulas elípticas a raramente lanceoladas (ovadas em $Q$. glazovii). É comumente conhecida como "mangue-tatarema" (Oliveira 1110 - UFP).

Material examinado: ALAGOAS: Flexeiras, fazenda Triunfo, 16.VIII.1968, fl., M.T. Monteiro 22706 (IPA, HST). Ibateguara, Coimbra, Grotão do Dudé, 25.IX.2002, fr., M. Oliveira $1110 \&$ A.A. Grilo (IPA, UFP). Grotão do Varjão, 25.I.2003, M.Oliveira \& A.A. Grilo 1267 (UFP). PERNAMBUCO: Igarassu, Usina São José, Mata da Piedade, 16.XII.2009, fr., J.A.N. Souza et al. 590 (UFP).

4. Quiina glazovii Engl. in Mart., Eichler \& Urb., Fl. bras. 12(1): 482; tab. 109. $1888 . \quad$ Fig. 1f-g Arvoretas a árvores androdióicas, com 5-15 m alt. e registro unicamente para Alagoas e Bahia. Segundo Reitz $(1965,1972)$ e Souza \& Bianchini (2001, 2007), ocorre também no Paraná, Rio de Janeiro, São Paulo e Santa Catarina, entre 50 e $450 \mathrm{~m}$ de altitude. É endêmica da Mata Atlântica e possivelmente ocorra também no Espírito Santo.

Quiina glazovii possui folhas e ramos jovens glabrescentes, folhas com 16-30 cm compr., margem revoluta, nervuras intersecundárias conspícuas e estípulas ovadas, foliáceas, glabras; inflorescência com 12-20 cm compr., composta por flores cremeamareladas a amarelo-intensas e odoríferas; e baga elipsóide. É facilmente reconhecida pelo formato oval das estípulas interpeciolares (filiformes a lineares em $Q$. cruegeriana e elípticas a raramente lanceoladas em $Q$. florida), além das folhas glabrescentes e inflorescências atingindo até $20 \mathrm{~cm}$ compr. Segundo Reitz (1972), os frutos são comestíveis e localmente conhecidos como "carobrano".

Material examinado: ALAGOAS: Maceió, fl., A. Uchoa 28 (RB). São Jorge da Laje, Usina Serra Grande, 28.IV.2001, M.S.Lopes \& M.R.S. Pietrobom 141 (UFP). BAHIA: Jussari, Serra do Teimoso, 7.II.1998, fl., W.W. Thomas et al. 11727 (CEPEC, NY). Itacaré, 3.IX.1970, fl., T.S. Santos 1072 (CEPEC, RB). Prado, BA-001 45 km de Alcobaça, 
19.III.1938, fr., S.A. Mori et al. 9731 (CEPEC). Ubaitaba, Ibirapitanga, 1.IX.1970, fl., T.S. Santos 1053 (CEPEC, IPA). Uruçuca, estrada para Serra Grande, 1-12.VII.1991, W.W. Thomas et al. 8654 (CEPEC, MO, NY).

\section{Agradecimentos}

Os autores agradecem aos curadores dos herbários visitados; aos assessores ad hoc, as valiosas contribuições; à Regina Carvalho, a alta qualidade das ilustrações botânicas; e, aos integrantes do laboratório de Morfo-Taxonomia Vegetal - UFPE, o constante auxílio.

\section{Referências Bibliográficas}

Barbosa, M.R.V.; Sothers, C.; Mayo, S.; Gamarra-Rojas, C.F.L. \& Mesquita, A.C. (orgs.). 2006. Checklist das plantas do Nordeste brasileiro: Angiospermas e gymnospermas. Ministério de Ciência e Tecnologia, Brasília. 156p.

Barreto, R.C.; Viana, A.M. B. \& Oliveira, J.B.S. 2006. Fanerógamas. In: Pôrto, K.C.; Almeida-Cortêz, J.S. \& Tabarelli, M. (orgs.). Diversidade biológica e conservação da floresta atlântica ao norte do rio São Francisco. Ministério do Meio Ambiente, Brasília. Pp. 175-190, 339-346.

Barroso, G.M.; Guimarães, E.F.; Ichaso, C.L.F.; Costa, C.G. \& Peixoto, A.L. 1978. Sistemática de angiospermas do Brasil. Vol. 1. $1^{\mathrm{a}}$ ed. LCT/EDUSP, São Paulo. 139p.

Costa, M.A.S. \& Amaral, M.C. 1999. Quiinaceae. In: Ribeiro, J.E.L.S.; Hopkins, M.J.G.; Vicentini, A.; Sothers, C.A.; Costa, M.A. S.; Brito, J.M.; Souza, M.A.D.; Martins, L.H.P.;Lohmann,L.G.; Assunção,P.A.C.L.;Pereira,E.C.; Silva, C.F.; Mesquita, M.R. \& Procópio, L.C. (orgs.). Flora da Reserva Ducke. INPA, Manaus. Pp. 241-243.

Engler, A. 1888. Guttiferae et Quiinaceae. In: Martius, C.F.P.; Eichler, A.W. \& Urban, I. (eds.). Flora brasiliensis. Frid. Fleischer, Leipzig. Vol. 12, pars 1. Pp. 381-492.

Ferraz, E.M.N. \& Rodal, M.J.N. 2006. Caracterização fisionômica-estrutural de um remanescente de floresta Ombrófila Montana de Pernambuco, Brasil. Acta Botanica Brasilica 20: 911-926.

Ferraz, E.M.N. \& Rodal, M.J.N. 2008. Floristic characterization of a remnant ombrophilous montane forest at São Vicente Férrer, Pernambuco, Brazil. In: Thomas, W.W. (ed.). The Atlantic coastal forest of Northeastern Brazil. Memoirs of the New York Botanical Garden 100: 67-146.

Fiaschi, P. \& Pirani, J.R. 2009. Review of plant biogeographic studies in Brazil. Journal of Systematics and Evolution 47: 477-496.
Grilo, A.A.; Oliveira, M.A. \& Tabarelli, M. 2006. Árvores. In: Pôrto, K.C.; Almeida-Cortêz, J.S. \& Tabarelli, M. (orgs.). Diversidade biológica e conservação da floresta atlântica ao norte do rio São Francisco. Ministério do Meio Ambiente, Brasília. Pp. 191-218, 347-349.

Reitz, R. 1965. Quiinaceae. In: Reitz, R. (ed.). Flora ilustrada catarinense. Parte I, fasc. QUII. Herbário Barbosa Rodrigues, Itajaí. Pp. 1-8.

Reitz, R. 1972. Quiinaceae. In: Reitz, R. \& Klein, R. (eds.). Flórula da Ilha de Santa Catarina. FISC, Florianópolis. Pp. 1-9.

Rocha, A.E.S. \& Secco, R.S. 2004. Uma sinopse de Lacunaria Ducke. Acta Amazonica 34: 425-433.

Schneider, J.V. \& Zizka, G. 2004. Quiinaceae. In: Steyermark, J.; Berry, P.E. \& Holst, B.K. (eds.). Flora of the Venezuelan Guayana. Vol. 8. Missouri Botanical Garden Press, Saint Louis. Pp. 393-407.

Schneider, J.V.; Swenson, U. \& Zizka, G. 2002. Phylogenetic reconstruction of the neotropical family Quiinaceae (Malpighiales) based on morphology with remarks on the evolution of an androdioecious sex distribution. Annals of the Missouri Botanical Gardens 89: 64-76.

Souza, F.O. \& Bianchini, R. 2001. Quiinaceae. In: Melo, M.M.R.F.; Barros, F.; Wanderley, M.G.L.; Kirizawa, M.; Jung-Mendaçolli, S. L. \& Chiea, S.A.C. (eds.). Flora fanerogâmica da Ilha do Cardoso. Vol. 8. Instituto de Botânica, São Paulo. Pp. 25-28.

Souza, F.O. \& Bianchini, R.S. 2007. Quiinaceae. In: Wanderley, M.G.L.; Shepherd, G.J.; Melhem, T.S.A. \& Giulietti, A.M. (ed.). Flora fanerogâmica do estado de São Paulo. Instituto de Botânica, São Paulo.Vol. 5. Pp. 255-258.

Souza, V.C. \& Lorenzi, H. 2008. Botânica sistemática: guia ilustrado para identificação das famílias de fanerógamas nativas e exóticas no Brasil, baseado na APG II. $2^{\mathrm{a}}$ ed. Instituto Plantarum, Nova Odessa. 704p.

Stevens, P.F. 2001 [onwards]. Angiosperm Phylogeny Website. Disponível em: <http://www.mobot.org/ MOBOT/research/APweb/>. Acesso em 3 setembro 2009.

Thiers, B. 2009 [continuously updated]. Index Herbariorum: A global directory of public herbaria and associated staff. New York Botanical Garden's Virtual Herbarium. Disponível em: <http://sweetgum. nybg.org/ih/>. Acesso em 3 setembro 2009.

Zizka, G. \& Schneider, J. V. 2004. Quiinaceae. In: Smith, N.; Mori, S. A.; Henderson, A.; Stevenson, D. Wm. \& Heald, S. V. (eds.). Flowering plants of the Neotropics. Princeton University Press, Princeton. Pp. 317-318. 
Lista de Exsicatas

Alves-Araújo, A. 1127 (2). Andrade-Lima, D. $67-9037$ (2), 67-9039 (2), 67-9038 (2), 68-45 (2), 69-5530 (2), $73-7311$ (2). Castro, A. J. s/n (EAC - 4122) (2). Ferraz, E. M. N. 609 (2); Belém, R. P. 3511 (1). Laurênio, A. 822 (2). Leal, J. 28 (2); 41 (2). Lira, O.

C. 68-243 (2). Lopes, M. S. 141 (4). Lyra-Lemos, R. P. 2781 (2). Martinelli, G. 9636 (1). Monteiro, M. T. 22706 (3). Mori, S. A. 9731 (4), 10637 (1), 13043 (1). Oliveira, J. B. S. 17 (2). Oliveira, M.1110 (3); 1267 (3), 1303 (2). Pires, J. M. $62-4042$ (2). Santos, T. S. 316 (1), 1053 (4), 1072 (4). Souza, J. A. N. 590 (3). Thomas, W. W. 8654 (4), 11727 (4). Uchoa, A. 28 (4). Viana, A. 187 (2). 